Research Article

\title{
Plantago major L. Extracts Reduce Blood Glucose in Streptozotocin-Induced Diabetic Mice
}

\author{
Do Thi Viet Huong $\mathbb{D}^{1},{ }^{1}$ Phan Minh Giang $\left(\mathbb{D},{ }^{1}\right.$ Nguyen Hoang Yen ${ }^{(D)}{ }^{1}$ \\ and Son Tien Nguyen $\mathbb{i D}^{2}$ \\ ${ }^{1}$ Faculty of Chemistry, VNU University of Science, Vietnam National University, Hanoi, Vietnam \\ ${ }^{2}$ Department of Rheumatology and Endocrinology, Vietnam Military Medical University, Hanoi, Vietnam
}

Correspondence should be addressed to Do Thi Viet Huong; dothiviethuong@gmail.com

Received 31 October 2020; Revised 10 April 2021; Accepted 26 May 2021; Published 7 June 2021

Academic Editor: Ajaya Kumar Singh

Copyright (c) 2021 Do Thi Viet Huong et al. This is an open access article distributed under the Creative Commons Attribution License, which permits unrestricted use, distribution, and reproduction in any medium, provided the original work is properly cited.

\begin{abstract}
Plantago major L. (P. major L.) is a perennial plant belonging to the family Plantaginaceae. It has been used as a folk remedy for diabetes in Europe and Asia. However, the biologically active constituents responsible for the antidiabetic effects have not been reported. The objectives of this study aimed at determining the chemical components of Plantago major L. and evaluating the antidiabetic activity of the extracts using streptozotocin- (STZ-) induced diabetic mice. In this study, the Swiss mice were fed a high-fat diet to gain weight before STZ injections to induce diabetic conditions. The STZ-induced diabetic mice were orally treated with $P$. major L. extracts. The blood glucose test results from the treated diabetic mice and nontreated diabetic mice were compared. We found that a 15-day treatment with EP6 extract from P. major L. at a dose of $400 \mathrm{mg} / \mathrm{kg}$ could reduce the blood glucose level to the same level as a 15-day treatment with glucophage at a dose of $70 \mathrm{mg} / \mathrm{kg}$. The major chemical components and structural characterization of EP6 extract were also reported. AST (aspartate transferase) and ALT (alanine aminotransferase) indicators of liver damage were measured in the treated and nontreated diabetic mice to give an overall view of the antidiabetic effect of $P$. major L. extracts.
\end{abstract}

\section{Introduction}

Diabetes mellitus, commonly known as diabetes, is a metabolic disorder causing high blood glucose and can lead to serious complications such as cardiovascular disease, stroke, chronic kidney disease, and nerve and vision damage [1]. There are two types of diabetes: type 1 diabetes results from the pancreas's disability to make enough insulin due to the loss of beta cells. Patients who suffer from type 1 diabetes are subjected to life-long insulin supplement therapy and are required to monitor blood insulin level regularly; type 2 diabetes often happens in overweight individuals, starting with cells that stop responding to insulin properly and possibly progressing to insulin deficiency. Among the two conditions, type 2 diabetes is considered to be more manageable and reversible by treatments with insulin sensitizers of biguanides, such as metformin, phenformin, and buformin, or thiazolidinediones, such as rosiglitazone, pioglitazone, and troglitazone, or Lyn kinase activators, such as tolimidone. However, these drugs have been linked to increased risks of lactic acidosis and liver damage [2-5].

Nature has been a generous source of bioactives for medicinal chemistry. P. major L. is a perennial plant populated in Europe and Asia. It has been used by the locals for the treatment of diabetes and other illnesses. Studies of chemical compositions of $P$. major $\mathrm{L}$. reveal that it contains flavonoids, terpenoids, and phenolic [6]. Those are phytochemicals that are produced by the plant through secondary metabolism and can possibly possess biological activities that serve as the plants' defense against competitors, predators, or pathogens. To our best knowledge, studies of the antidiabetic effect of $P$. major $\mathrm{L}$. in type 2 diabetes treatment are limited. A few studies have shown that the extracts from $P$. major L. were effective in improving blood glucose in type 2 diabetic mice [7-9]. These studies show that the antidiabetic activity test using STZ-induced diabetic 
mice at a dose of $1000 \mathrm{mg}$ alcoholic extract/kg rat body weight of $P$. major $\mathrm{L}$. reduced the blood glucose to a comparable level of the control group at the $5^{\text {th }}$ day until the $14^{\text {th }}$ day of the study; oral administration of $P$. major L. extract at the doses of $600 \mathrm{mg}$ aqueous extract $/ \mathrm{kg}$ rat b.w. for alloxan monohydrate-induced diabetic rat significantly decreases the blood glucose level and $500 \mathrm{mg}$ methanol extract/kg body weight promotes glucose uptake in mice with efficient insulin-secreting pancreas. However, thorough isolation and chemical characterization of the extract components of $P$. major L. and the determination of bioactives responsible for antidiabetic effects have not been reported. Thus, we carried out a thorough extraction and then column chromatography isolation and structural determination of $P$. major L. extracts by NMR (nuclear magnetic resonance) spectroscopy. To test the glycemic control activity of $P$. major $L$. extracts, an in vivo experiment on mice was performed starting with preparing obese mice, inducing diabetes with STZ, followed by the treatment with $P$. major L. extracts. The antihyperglycemic effect was determined via a blood glucose test. Importantly, to identify any negative effects of the extracts on liver function and biosafety of the extract, liver lipid and enzymes profile, including cholesterol, TG (triglyceride), AST, and ALT were measured. Furthermore, liver enzymes are associated with the diabetic nonalcoholic liver fatty disease. Our studies show that $P$. major L. extracts were capable of reducing elevated liver enzymes in diabetic mice compared to the control group. Hence, this paper provides important information to fill the knowledge gaps in $P$. major L. antihyperglycemic activity research: chemical structural determination of the antihyperglycemic components of $P$. major L. and in vivo assessment of the safety of P. major $\mathrm{L}$. in treatment of the hyperglycemic condition in mice.

\section{Materials and Methods}

2.1. Plant Source. The plant sample was grown and harvested in September 2018 in Hoai Duc province, Vietnam. The plant was identified as P. major L. by Dr. Trieu Anh Trung, Faculty of Biology, Hanoi National University of Education. All parts of P. major L. plant were washed with water to remove soil and dried in the shade until drained. Fresh plant samples were dried at 40 degrees until complete dryness. The dry sample is ground into a powder using a homemade blender.

\subsection{Extract Preparation and Compounds Isolation.} P. major L. $(5 \mathrm{~kg})$ was immersed in ethyl acetate at room temperature. After 7 days, the extract was collected, and the residue was repeatedly extracted two times. The combined extract was concentrated under vacuum to yield a solid EP $(306 \mathrm{~g})$. The extract was subjected to silica gel column chromatography eluting with a gradient system of $n$-hexane/ ethyl acetate $(20: 1 \longrightarrow 0: 1)$ to provide 6 fractions (EP1EP6). Fraction EP3 was further purified by silica gel column chromatography eluting with a gradient system of $n$-hexane/ acetone $(20: 1)$ to provide 4 fractions (EP3.1-EP3.4).
Fraction EP3.2 was further purified by silica gel column chromatography eluted with a gradient system of $n$-hexane: ethyl acetate $(4: 1)$ to give two purified compounds EPL1 $(20 \mathrm{mg})$ and EPL2 $(13 \mathrm{mg})$. Fraction EP4 was further purified with chloroform/methanol $(10: 1)$ to provide 4 fractions (EP4.1-EP4.4). Fractions EP4.2 and EP4.3 were purified by high-performance liquid chromatography on the RP-18 CC column, eluted by chloroform/methanol/water $(10: 5: 1)$ to give two purified compounds EPL3 $(10 \mathrm{mg}$ ) and EPL4 $(10 \mathrm{mg})$, respectively. Fractions EP4.4 and EP6 were also purified on high-performance liquid chromatography RP-18 column, eluted by chloroform/methanol/water $(14: 6: 1)$ to give purified compounds of EPL5 $(15 \mathrm{mg})$, EPL6 $(8 \mathrm{mg})$, EPL7 $(10 \mathrm{mg})$, and EPL8 $(8 \mathrm{mg})$. The separation procedure from the ethyl acetate $P$. major L. extract is described in Figure 1. The structures of purified compounds were determined on the basis of ${ }^{1} \mathrm{H}$ NMR and ${ }^{13} \mathrm{C}$ NMR spectral data by comparison with literature values [10-14]. Compounds EPL1-8 were assigned as lupeol (EPL1), friedelan 3one (EPL2), quassin (EPL3), 18-hydroxy quassin (EPL4), (+)-catechin (EPL5), epicatechin (EPL6), 7-O-methylapigenin (EPL7), and 7, 4'-O-dimethylapigenin (EPL8), as shown in Figure 2.

\subsection{Design of the In Vivo Experiment}

2.3.1. Animals and Induction of Diabetes in Obese Mice. Swiss mice weighing $18-22 \mathrm{~g}$ were provided from the $\mathrm{Na}$ tional Institute of Hygiene and Epidemiology, Hanoi, Vietnam, and were humanely treated in accordance with the Ministry of Health Guidelines for the Care and Use of Laboratory Animals. The protocols were approved by the Ethics Committee of VNU Pharmaceutical Chemistry Laboratory (permit number: A 2019-0033). Animals were kept 10 mice per group of a total of 8 groups and divided into two experiments: the high-fat diet-fed (HFD) group with 70 animals (groups 1-7) and the normal control with 10 animals (group NC). The animals were housed in clean metabolic cages placed in a well-ventilated house with an optimum condition (temperature: $25 \pm 2^{\circ} \mathrm{C}$; photoperiod: $12 \mathrm{~h}$ natural light and $12 \mathrm{~h}$ dark; and humidity: $45-50 \%)$. The cleaning of the cages was done on a daily basis. The HFD group were provided with a high-fat diet consisting of carbohydrate $(40 \%)$, fat $(30 \%)$, protein $(20 \%)$, and other vitamins and minerals (5\%). The NC mice were given a standardized diet consisting of protein carbohydrate (53\%), protein (20\%), and fat (6\%). Body weight was recorded weekly. After 8 weeks, HFD mice show a significant increase in body weight $(158 \%-204 \%)$ compared with NC mice (78\%), as shown in Table 1.

Mice whose body weights were over $50 \mathrm{~g}$ in the HFD groups (cages 1-6) were injected with STZ $(120 \mathrm{mg} / \mathrm{kg} \mathrm{bw})$. The high-fat-fed NCF mice (average body weight $33.52 \mathrm{~g}$ ) were added with physiological saline only. The blood glucose level in both STZ-induced diabetic group and control group was measured by the One Touch Ultra device before the STZ injection and at $48 \mathrm{~h}, 72 \mathrm{~h}, 5$ days, 7 days, and 10 days after the injection $[15,16]$. The results are tabulated in Table 2. 


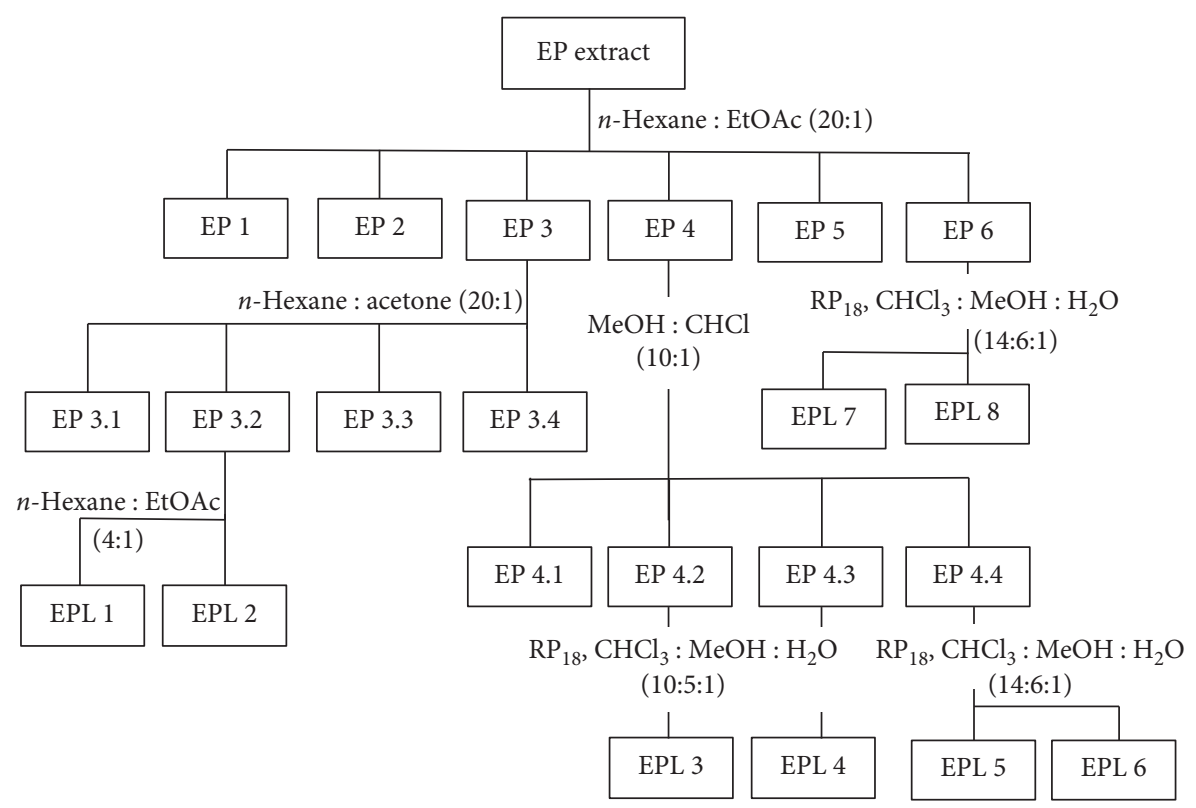

Figure 1: The separation procedure of the ethyl acetate extract.

After 10 days of STZ injection, mice with blood glucose levels $>18 \mathrm{mmol} / \mathrm{L}$ were considered type 2 diabetic [17]. The diabetic animals were subjected to further treatment (Table 3 ). Diabetic mice in Cage 1, the normal control diabetic group, are only treated with saline solution. Diabetic mice in Cage 2: the positive control group, are treated with Glucophage $(70 \mathrm{mg} / \mathrm{kg})$. The diabetic mice in Cages 3, 4, 5, and 6 received $400 \mathrm{mg} / \mathrm{kg}$ of extractions EP, EP3, EP4, and EP6, accordingly.

Mice in those cages were given the treatments at exactly 8 a.m. daily. The blood glucose level in each cage was measured before and at $2 \mathrm{~h}, 4 \mathrm{~h}, 8 \mathrm{~h}, 3$ days, and 15 days after the oral intake. The results are shown in Figure 3 and Table 4.

2.4. Determination of Blood Glucose Level and Biochemical Parameters. The venous blood of mice was collected from the tail for measuring the blood glucose levels using a glucose monitoring system (One Touch brand). Four groups from obese mice diabetic group treated with EP6 (Cage 6), obese mice diabetic with no treatment (Cage 1), high-fat-fed mice (NCf), and normal mice (NC) were chosen, and their blood was collected and centrifuged at 3,000 rpm for 5 minutes. The separation serum was used for the biochemistry assay. The serum parameters: cholesterol level and triglycerides level, alanine aminotransferase level (ALT), and aspartate transaminase level (AST) were estimated using QuickDetect Total cholesterol/Triglyceride/AST/ALT (Rat) ELISA Kit (Ray Biotech). Consequently, these blood samples were centrifuged at a velocity of $3000 \mathrm{rpm}$ for 15 minutes. Sera were then analyzed with commercially available kits (Sandwich method) according to the manufacturer's protocol by ELISA apparatus (Photometer $5010 \mathrm{V5}^{+}$, Robert Riele, Germany). Changes in color were checked at a wavelength of $450 \mathrm{~nm}$. According to manufacture information, both the intra-assay and interassay coefficients of variation were below $12 \%$.
2.5. Statistics. Data are presented as mean \pm SD. Statistical significance was assessed by group comparison with the use of one-way ANOVA followed by the Tukey-Kramer test. Significance was accepted at $p<0.05$. All data were analyzed with the SPSS version 26 (64-bit edition) for Windows (SPSS Inc., Chicago, IL).

\section{Results}

3.1. Identification of Compounds from P. major L. Extract. EPL1, lupeol, white crystals, ${ }^{1} \mathrm{H} \mathrm{NMR}\left(\mathrm{CDCl}_{3}, 500 \mathrm{MHz}\right), \delta_{\mathrm{H}}$ (ppm): $3.34(1 \mathrm{H}, \mathrm{dd}, J=11.5,5.0 \mathrm{~Hz}, \mathrm{H}-3), 2.08(1 \mathrm{H}, \mathrm{dd}$, $J=11.0,6.0 \mathrm{~Hz}, \mathrm{H}-19), 0.80$ (3H, s, H-23), $0.76(3 \mathrm{H}, \mathrm{s}, \mathrm{H}-24)$, $0.84(3 \mathrm{H}, \mathrm{s}, \mathrm{H}-25), 0.90(3 \mathrm{H}, \mathrm{s}, \mathrm{H}-26), 0.91(3 \mathrm{H}, \mathrm{s}, \mathrm{H}-27), 0.70$ (3H, s, H-28), $4.92(2 \mathrm{H}, \mathrm{s}, \mathrm{H}-29), 1.79(3 \mathrm{H}, \mathrm{s}, \mathrm{H}-30) .{ }^{13} \mathrm{C}$ NMR $\left(\mathrm{CDCl}_{3}, 125 \mathrm{MHz}\right), \delta_{\mathrm{C}}(\mathrm{ppm}): 38.40(\mathrm{C}-1), 27.45$ (C2), 78.03 (C-3), 38.90 (C-4), 55.26 (C-5), 17.35 (C-6), 35.24 (C-7), 40.90 (C-8), 50.35 (C-9), 37.51 (C-10), 21.97 (C-11), 25.41 (C-12), 38.01 (C-13), 43.01 (C-14), 26.57 (C-15), 35.59 (C-16), 42.98 (C-17), 48.40 (C-18), 151.03 (C-20), 29.7 (C21), 39.03 (C-22), 23.94 (C-23), 23.83 (C-24), 15.99 (C-25), 19.01 (C-26), 14.98 (C-27), 18.63 (C-28), 109.33 (C-29), 21.37 (C-30).

EPL2, friedelan 3-one, white powder, ${ }^{1} \mathrm{H}$ NMR $\left(\mathrm{CDCl}_{3}\right.$, $500 \mathrm{MHz}), \delta_{\mathrm{H}}(\mathrm{ppm}): 1.48(2 \mathrm{H}, \mathrm{m}, \mathrm{H}-1), 2.32(2 \mathrm{H}, \mathrm{dd}, J=7.0$; $13.0 \mathrm{~Hz}, \mathrm{H}-2), 2.14(1 \mathrm{H}, \mathrm{br} \mathrm{d}, J=7.0 \mathrm{~Hz}, \mathrm{H}-4), 1.37 ; 1.56(2 \mathrm{H}$, m, H-6), 1.36; $1.70(2 \mathrm{H}, \mathrm{m}, \mathrm{H}-7), 1.00(1 \mathrm{H}, \mathrm{m}, \mathrm{H}-8), 0.99(1 \mathrm{H}$, $\mathrm{m}, \mathrm{H}-10), 1.25 ; 1.60$ (2H, m, H-11), 1.26; 1.61 (2H, m, H-12), $1.25 ; 1.61(2 \mathrm{H}, \mathrm{m}, \mathrm{H}-15), 1.25 ; 1.59(2 \mathrm{H}, \mathrm{m}, \mathrm{H}-16), 1.09(1 \mathrm{H}$, m, H-18), 1.29; 1.08 (2H, m, H-19), 1.56; 0.96 (2H, m, H-21), 1.66; $1.31(2 \mathrm{H}, \mathrm{m}, \mathrm{H}-22), 1.08$ (3H, d, $J=7,0 \mathrm{~Hz}, \mathrm{H}-23), 1.22$ (3H, s, H-24), 0.84 (3H, s, H-25), 0.98 (3H, s, H-26), 0.98 (3H, s, H-27), 1.01 (3H, s, H-28), 0.85 (3H, s, H-29), 1.01 $(3 \mathrm{H}, \mathrm{s}, \mathrm{H}-30) .{ }^{13} \mathrm{C}$ NMR $\left(\mathrm{CDCl}_{3}, 125 \mathrm{MHz}\right), \delta_{\mathrm{C}}(\mathrm{ppm}): 21.98$ (C-1), 41.68 (C-2), 213.72 (C-3), 59.01 (C-4), 42.21 (C-5), 40.97 (C-6), 18.49 (C-7), 51.94 (C-8), 37.79 (C-9), 59.15 (C- 


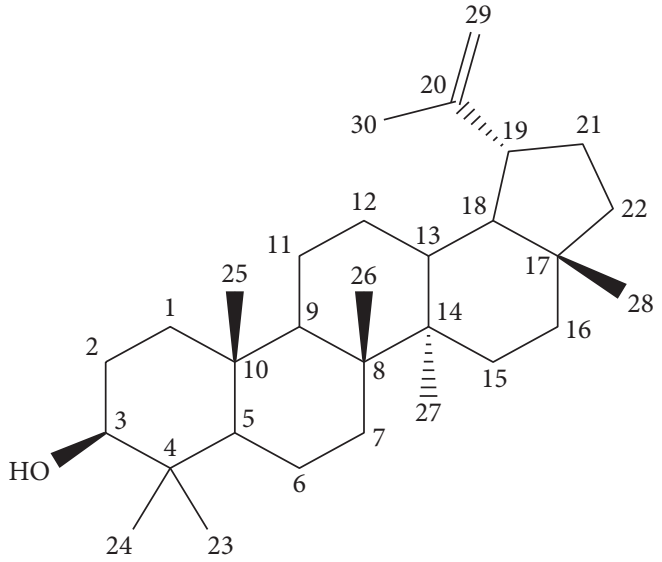

(a)

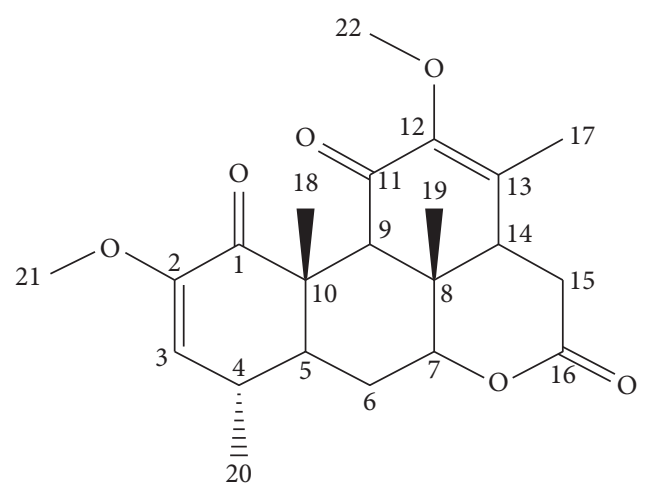

(c)<smiles>Oc1cc(O)c2c(c1)O[C@H](c1ccc(O)c(O)c1)[C@H](O)C2</smiles>

(e)<smiles>O=c1cc(-c2ccc(O)cc2)oc2cc(OI)cc(O)c12</smiles>

(g)

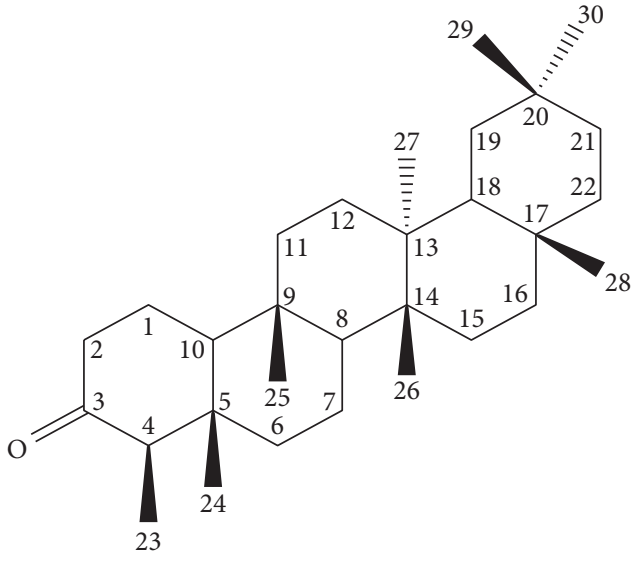

(b)

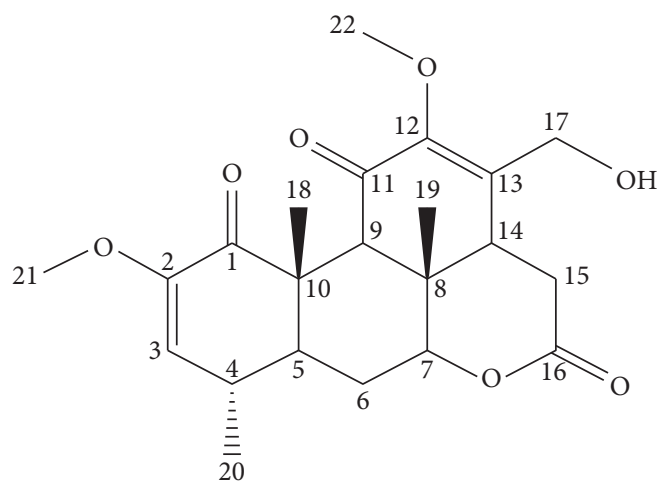

(d)<smiles>Oc1cc(O)c2c(c1)O[C@H](c1ccc(O)c(O)c1)[C@H](O)C2</smiles>

(f)<smiles>O=c1cc(-c2ccc(O)cc2)oc2cc(OI)cc(O)c12</smiles>

(h)

Figure 2: Compounds isolated from the ethyl acetate extract. (a) EPL1: lupeol, (b) EPL2: friedelan 3-one, (c) EPL3: quassin, (d) EPL4: 17hydroxy quassin, (e) EPL5: (+)-catechin, (f) EPL6: epicatechin, (g) ELP7: 7-O-methylapigenin, and (h) EPL8: 7, 4'-O-dimethylapigenin. 
TABLE 1: Body weight of mice in the high-fat diet-fed group (HFD) and the normal control group (NC) group before and after 8-week diet.

\begin{tabular}{lccc}
\hline Group & Week 0 $(\mathrm{g})$ & Week 8 $(\mathrm{g})$ & \% increase \\
\hline 1 & 18.91 & 53.72 & 184.08 \\
2 & 19.22 & 52.31 & 172.16 \\
3 & 18.73 & 50.24 & 168.23 \\
4 & 18.96 & 54.85 & 189.29 \\
5 & 19.92 & 51.46 & 158.33 \\
6 & 18.15 & 55.24 & 204.35 \\
7 & 18.97 & 51.92 & 173.69 \\
NC & 18.78 & 33.52 & 78.48 \\
\hline
\end{tabular}

TABLE 2: Blood glucose level of STZ-induced diabetic group and control group (saline added).

\begin{tabular}{lcc}
\hline $\begin{array}{l}\text { Group } \\
\text { time }\end{array}$ & $\begin{array}{c}\text { Control group (saline) } \\
(\mathrm{mmol} / \mathrm{l})\end{array}$ & $\begin{array}{c}\text { STZ-induced diabetic group } \\
(\mathrm{mmol} / \mathrm{l})\end{array}$ \\
\hline $0 \mathrm{~h}$ & $6.4 \pm 0.7$ & $10.1 \pm 0.1$ \\
$48 \mathrm{~h}$ & $6.2 \pm 0.3$ & $16.9 \pm 0.8^{*}$ \\
$72 \mathrm{~h}$ & $5.9 \pm 0.6$ & $20.2 \pm 0.7^{*}$ \\
5 days & $5.8 \pm 1.2$ & $21.7 \pm 0.2^{*}$ \\
7 days & $5.3 \pm 1$ & $25.8 \pm 4.2^{*}$ \\
10 days & $5.7 \pm 0.8$ & $24.4 \pm 0.6^{*}$ \\
\hline
\end{tabular}

Significantly different from blood glucose before STZ injection $(t=0)$ $\left({ }^{*} p<0.05\right)$.

TABle 3: Treatment and dose for STZ-induced diabetic mice in Cages 1-6.

\begin{tabular}{lcc}
\hline Cage & Treatment & Dose \\
\hline 1 (normal control diabetic) & Saline $0.9 \%$ & $10 \mathrm{~mL} / \mathrm{kg}$ \\
2 (positive control) & Glucophage & $70 \mathrm{mg} / \mathrm{kg}$ \\
3 & EP & $400 \mathrm{mg} / \mathrm{kg}$ \\
4 & EP3 & $400 \mathrm{mg} / \mathrm{kg}$ \\
5 & EP4 & $400 \mathrm{mg} / \mathrm{kg}$ \\
6 & EP6 & $400 \mathrm{mg} / \mathrm{kg}$ \\
\hline
\end{tabular}

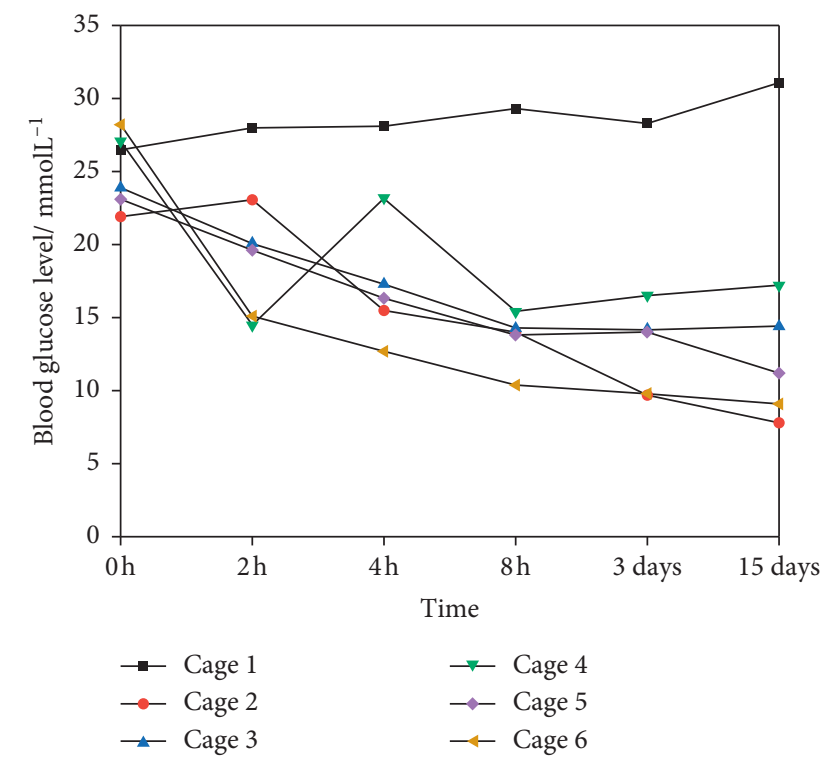

FIgURE 3: Blood glucose level of STZ-induced diabetic mice after treatments.
10), 35.08 (C-11), 30.05 (C-12), 39.99 (C-13), 40.97 (C-14), 31.02 (C-15), 36.37 (C-16), 31.05 (C-17), 42.80 (C-18), 36.01 (C-19), 30.52 (C-20), 33.78 (C-21), 38.10 (C-22), 7.57 (C-23), 13.51 (C-24), 15.99 (C-25), 19.04 (C-26), 19.00 (C-27), 31.88 (C-28), 31.80 (C-29), 31.76 (C-30).

EPL3: quassin, white powder, ${ }^{1} \mathrm{H}$ NMR $\left(\mathrm{CDCl}_{3}\right.$, $500 \mathrm{MHz}), \delta_{\mathrm{H}}(\mathrm{ppm}): 5.51(1 \mathrm{H}, \mathrm{d}, J=2,5 \mathrm{~Hz}, \mathrm{H}-3), 2.29(1 \mathrm{H}$, dd, $J=2,5 ; 7,9 \mathrm{~Hz}, \mathrm{H}-4), 1.99(1 \mathrm{H}, \mathrm{dd}, J=3.0 ; 14.0, \mathrm{H}-5), 1.71$; 1.49 (2H, m, H-6), 4.48 (1H, dd, J=1.0; $3.0 \mathrm{~Hz}, \mathrm{H}-7), 2.29$ (1H, s, H-9), 2.34 (1H, dd, $J=7.0 ; 11.5 \mathrm{~Hz}, \mathrm{H}-14), 2.59 ; 2.37$ $(2 \mathrm{H}, \mathrm{dd}, J=7,0 ; 11,5 \mathrm{~Hz}, \mathrm{H}-15), 5.44(3 \mathrm{H}, \mathrm{s}, \mathrm{H}-17), 1.28(3 \mathrm{H}$, s, H-18), 1.08 (3H, s, H-19), 1.09 (3H, d, H-20), 3.78 (3H, s, $\mathrm{H}-21), 3.87(3 \mathrm{H}, \mathrm{s}, \mathrm{H}-22) .{ }^{13} \mathrm{C} \mathrm{NMR}\left(\mathrm{CDCl}_{3}, 125 \mathrm{MHz}\right), \delta_{\mathrm{C}}$ (ppm): 198.8 (C-1), 148.2 (C-2), 118.4 (C-3), 32.2 (C-4), 44.0 (C-5), 27.9 (C-6), 82.0 (C-7), 40.1 (C-8), 45.7 (C-9), 46.6 (C10), 195.0 (C-11), 147.8 (C-12), 120.2 (C-13), 39.9 (C-14), 31.7 (C-15), 169.1 (C-16), 14.4 (C-17), 13.0 (C-18), 23.0 (C19), 20.1 (C-20), 54.9 (C-21), 57.6 (C-22).

EPL4: 17-hydroxy quassin, white powder, ${ }^{1} \mathrm{H}$ NMR $\left(\mathrm{CDCl}_{3}, 500 \mathrm{MHz}\right), \delta_{\mathrm{H}}(\mathrm{ppm}): 5.49(1 \mathrm{H}, \mathrm{d}, J=2.0 \mathrm{~Hz}, \mathrm{H}-3)$, $2.18(2 \mathrm{H}, \mathrm{m}, \mathrm{H}-4), 1.51(1 \mathrm{H}, \mathrm{dd}, J=2.5 ; 14.0 \mathrm{~Hz}, \mathrm{H}-5), 1.78$; 1.55 (2H, m, H-6), 4.51 (1H, dd, J=2.0; $5.0 \mathrm{~Hz}, \mathrm{H}-7), 2.75$ $(1 \mathrm{H}, \mathrm{s}, \mathrm{H}-9), 2.78(1 \mathrm{H}, \mathrm{dd}, J=7.0 ; 11.5 \mathrm{~Hz}, \mathrm{H}-14), 3.21(2 \mathrm{H}$, dd, $J=7.0 ; 18.5 \mathrm{~Hz}, \mathrm{H}-15), 4.03(2 \mathrm{H}, \mathrm{d}, J=14.5 \mathrm{~Hz}, \mathrm{H}-17)$, 1.27 (3H, s, H-18), 1.09 (3H, s, H-19), 1.03 (3H, d, J= 7.0 Hz, H-20), 3.88 (3H, s, H-21), 3.83 (3H, s, H-22), 10.19 (2H, s, $\mathrm{OH}-17) .{ }^{13} \mathrm{C} \mathrm{NMR}\left(\mathrm{CDCl}_{3}, 125 \mathrm{MHz}\right), \delta_{\mathrm{C}}(\mathrm{ppm}): 197.9(\mathrm{C}-$ 1), 148.0 (C-2), 116.6 (C-3), 31.1 (C-4), 43.7 (C-5), 27.9 (C6), 81.9 (C-7), 37.9 (C-8), 45.6 (C-9), 46.9(C-10), 191.6 (C11), 148.0 (C-12), 126.6 (C-13), 36.3 (C-14), 31.6 (C-15), 168.2 (C-16), 54.6 (C-17), 12.8 (C-18), 22.4 (C-19), 20.1 (C20), 54.8 (C-21), 58.0 (C-22).

EPL5: (+)-catechin, white powder, ${ }^{1} \mathrm{H}$ NMR $\left(\mathrm{CD}_{3} \mathrm{OD}\right.$, $500 \mathrm{MHz}), \delta_{\mathrm{H}}(\mathrm{ppm}): 4.88(1 \mathrm{H}, \mathrm{d}, J=7.5 \mathrm{~Hz}, \mathrm{H}-2), 4.89(1 \mathrm{H}$, m, H-3), 2.81; 2.56 (2H, dd, $J=16.0,8.5 \mathrm{~Hz}, \mathrm{H}-4), 5.87(1 \mathrm{H}$, d, $J=2.5 \mathrm{~Hz}, \mathrm{H}-6), 5.91(1 \mathrm{H}, \mathrm{d}, J=2.5 \mathrm{~Hz}, \mathrm{H}-8), 6.66(1 \mathrm{H}, \mathrm{d}$, $\left.J=1.5 \mathrm{~Hz}, \mathrm{H}-2^{\prime}\right), 6.64\left(1 \mathrm{H}, \mathrm{d}, J=8.0 \mathrm{~Hz}, \mathrm{H}-5^{\prime}\right), 6.64(1 \mathrm{H}, \mathrm{dd}$, $\left.J=8.0 ; 1,5 \mathrm{~Hz}, \mathrm{H}-6^{\prime}\right), 5.02(1 \mathrm{H}, \mathrm{s}, \mathrm{OH}-3), 9.60$ (1H, s, OH-5), $10.31(1 \mathrm{H}, \mathrm{s}, \mathrm{OH}-7), 9.39\left(1 \mathrm{H}, \mathrm{s}, \mathrm{OH}-3^{\prime}\right), 9.50\left(1 \mathrm{H}, \mathrm{s}, \mathrm{OH}-4^{\prime}\right)$. ${ }^{13} \mathrm{C} \mathrm{NMR}\left(C D_{3} \mathrm{OD}, 125 \mathrm{MHz}\right), \delta_{\mathrm{H}}(\mathrm{ppm}): 85.87$ (C-2), 67.73 (C-3), 28.51 (C-4), 157.28 (C-5), 95.29 (C-6), 157.58 (C-7), 95.08 (C-8), 157.09 (C-9), 100.01 (C-10), 131.58 (C-1'), $115.29\left(\mathrm{C}-2^{\prime}\right), 145.85\left(\mathrm{C}-3^{\prime}\right), 144.66\left(\mathrm{C}-4^{\prime}\right), 116.11\left(\mathrm{C}-5^{\prime}\right)$, $120.94\left(\mathrm{C}-6^{\prime}\right)$.

EPL6: epicatechin, white powder, ${ }^{1} \mathrm{H}$ NMR $\left(\mathrm{CD}_{3} \mathrm{OD}\right.$, $500 \mathrm{MHz}), \delta_{\mathrm{H}}(\mathrm{ppm}): 4.83(1 \mathrm{H}, \mathrm{d}, \mathrm{H}-2), 4.20(1 \mathrm{H}, \mathrm{m}, \mathrm{H}-3)$, $2.88(1 \mathrm{H}, \mathrm{dd}, J=12.0,2.5 \mathrm{~Hz}, \mathrm{H}-4), 5.96(1 \mathrm{H}, \mathrm{m}, \mathrm{H}-6), 6.99$ $\left(1 \mathrm{H}, \mathrm{d}, J=1.5 \mathrm{~Hz}, \mathrm{H}-2^{\prime}\right), 6.77\left(1 \mathrm{H}, \mathrm{m}, \mathrm{H}-5^{\prime}\right), 6.82(1 \mathrm{H}, \mathrm{m}$, H-6 $\left.{ }^{\prime}\right), 5.02$ (1H, s, OH-3), $9.68(1 \mathrm{H}, \mathrm{s}, \mathrm{OH}-5), 10.29(1 \mathrm{H}, \mathrm{s}$, $\mathrm{OH}-7), 9.48\left(1 \mathrm{H}, \mathrm{s}, \mathrm{OH}-3^{\prime}\right), 9.44\left(1 \mathrm{H}, \mathrm{s}, \mathrm{OH}-4^{\prime}\right) .{ }^{13} \mathrm{C} \mathrm{NMR}$ $\left(C D_{3} \mathrm{OD}, 125 \mathrm{MHz}\right), \delta_{\mathrm{C}}(\mathrm{ppm}): 79.8$ (C-2), 67.4 (C-3), 29.2 (C-4), 157.9 (C-5), 96.4 (C-6), 157.5 (C-7), 95.9 (C-8), 157.3 (C-9), 100.0 (C-10), $132.2\left(\mathrm{C}-1^{\prime}\right), 115.3\left(\mathrm{C}-2^{\prime}\right), 145.7\left(\mathrm{C}-3^{\prime}\right)$, $145.9\left(\mathrm{C}-4^{\prime}\right), 115.9\left(\mathrm{C}-5^{\prime}\right), 119.4\left(\mathrm{C}-6^{\prime}\right)$.

ELP7: 7-O-methylapigenin, white powder, ${ }^{1} \mathrm{H}$ NMR $\left(\mathrm{DMSO}_{\mathrm{d}}, 500 \mathrm{MHz}\right), \delta_{\mathrm{H}}(\mathrm{ppm}): 6.75(1 \mathrm{H}, \mathrm{s}, \mathrm{H}-3), 6.18(1 \mathrm{H}$, d, $J=2.4 \mathrm{~Hz}, \mathrm{H}-6), 6.77(1 \mathrm{H}, \mathrm{d}, J=2.4 \mathrm{~Hz}, \mathrm{H}-8), 7.56(1 \mathrm{H}, \mathrm{d}$, $\left.J=9.0 \mathrm{~Hz}, \mathrm{H}-2^{\prime}\right), 6.66\left(1 \mathrm{H}, \mathrm{d}, J=9.0 \mathrm{~Hz}, \mathrm{H}-3^{\prime}\right), 6.64(1 \mathrm{H}, \mathrm{d}$, $\left.J=9.0 \mathrm{~Hz}, \mathrm{H}-5^{\prime}\right), 7.48\left(1 \mathrm{H}, \mathrm{d}, J=9.0 \mathrm{~Hz}, \mathrm{H}-6^{\prime}\right), 13.47(1 \mathrm{H}, \mathrm{s}$, 
TABLE 4: Blood glucose level of STZ-induced diabetic mice after treatments.

\begin{tabular}{|c|c|c|c|c|c|c|c|}
\hline & $0 \mathrm{~h}$ & $2 \mathrm{~h}$ & $4 \mathrm{~h}$ & $8 \mathrm{~h}$ & 3 days & 15 days & $\%$ blood glucose decrease \\
\hline Cage 1: (NCd) $\mathrm{NaCl} 0,9 \%$ & $26.5 \pm 1.1$ & $28 \pm 1.8$ & $28.1 \pm 1.3$ & $29.3 \pm 1.9$ & $28.3 \pm 0.8$ & $31.1 \pm 1.1$ & 0 \\
\hline Cage 2: glucophage & $21.9 \pm 1.5$ & $23.1 \pm 1.8$ & $15.5 \pm 0.7$ & $14.1 \pm 3.1^{*}$ & $9.7 \pm 2.2^{*}$ & $7.8 \pm 1.5^{*}$ & 64.38 \\
\hline Cage 3: EP & $23.9 \pm 0.9$ & $20.1 \pm 2.3^{*}$ & $17.3 \pm 5.3^{*}$ & $14.3 \pm 2.1^{*}$ & $14.2 \pm 0.9^{*}$ & $14.4 \pm 0.8^{*}$ & 39.74 \\
\hline Cage 4: EP3 & $27.1 \pm 3.2$ & $14.5 \pm 2.1^{*}$ & $23.2 \pm 2.4^{*}$ & $15.4 \pm 0.3^{*}$ & $16.5 \pm 1.2^{*}$ & $17.2 \pm 4.2^{*}$ & 36.53 \\
\hline Cage 5: EP4 & $23.1 \pm 0.9$ & $19.6 \pm 2.3^{*}$ & $16.3 \pm 5.3^{*}$ & $13.8 \pm 2.1^{*}$ & $14.0 \pm 0.9^{*}$ & $11.2 \pm 0.8^{*}$ & 51.51 \\
\hline Cage 6: EP6 & $28.2 \pm 2.3$ & $15.1 \pm 0.9^{* *}$ & $12.7 \pm 1.5^{* *}$ & $10.4 \pm 5.3^{* *}$ & $9.8 \pm 2.3^{* *}$ & $9.1 \pm 1.9^{* *}$ & 67.73 \\
\hline
\end{tabular}

Significantly different from blood glucose level of the NC group taken at the same time $\left({ }^{*} p<0.05 ;{ }^{* *} p<0.01\right)$.

$\mathrm{OH}-5), 3.81\left(3 \mathrm{H}, \mathrm{s}, \mathrm{OCH}_{3}-7\right) .{ }^{13} \mathrm{C}$ NMR (DMSO-d 6 , $125 \mathrm{MHz}), \delta_{\mathrm{C}}$ (ppm): $163.8(\mathrm{C}-2), 104.5(\mathrm{C}-3), 182.6(\mathrm{C}-4)$, 167.1 (C-5), 97.9 (C-6), 167.2 (C-7), 92.6 (C-8), 159.0 (C-9), 104.1 (C-10), $122.9\left(\mathrm{C}-1^{\prime}\right), 130.0\left(\mathrm{C}-2^{\prime}\right), 116.1\left(\mathrm{C}-3^{\prime}\right), 159.3$ $\left(\mathrm{C}-4^{\prime}\right), 119.0\left(5^{\prime}\right), 129.9\left(\mathrm{C}-6^{\prime}\right), 55.9$ (C-11).

EPL8: 7, $4^{\prime}$-O-dimethylapigenin, white powder, ${ }^{1} \mathrm{H}$ NMR $\left(\mathrm{CD}_{3} \mathrm{OD}, 500 \mathrm{MHz}\right), \delta_{\mathrm{H}}(\mathrm{ppm}): 6.75(1 \mathrm{H}, \mathrm{s}, \mathrm{H}-3), 6.17(1 \mathrm{H}, \mathrm{d}$, $J=2.4 \mathrm{~Hz}, \mathrm{H}-6), 6.78(1 \mathrm{H}, \mathrm{d}, J=2.4 \mathrm{~Hz}, \mathrm{H}-8), 7.99(1 \mathrm{H}, \mathrm{d}$, $\left.J=7.0 \mathrm{~Hz}, \mathrm{H}-2^{\prime}\right), 7.03\left(1 \mathrm{H}, \mathrm{d}, J=7.0 \mathrm{~Hz}, \mathrm{H}-3^{\prime}\right), 7.03(1 \mathrm{H}, \mathrm{d}$, $\left.J=7.0 \mathrm{~Hz}, \mathrm{H}-5^{\prime}\right), 7.94\left(1 \mathrm{H}, \mathrm{d}, J=7.0 \mathrm{~Hz}, \mathrm{H}-6^{\prime}\right), 12.01(1 \mathrm{H}, \mathrm{s}$, $\mathrm{OH}-5), 3.79\left(3 \mathrm{H}, \mathrm{s}, \mathrm{OCH}_{3}-7\right), 3.79\left(3 \mathrm{H}, \mathrm{s}, \mathrm{OCH}_{3}-4^{\prime}\right) .{ }^{13} \mathrm{C}$ $\operatorname{NMR}\left(\mathrm{CD}_{3} \mathrm{OD}, 125 \mathrm{MHz}\right), \delta_{\mathrm{C}}(\mathrm{ppm}): 163.9$ (C-2), 104.5 (C3), 181.9 (C-4), 161.4 (C-5), 98.0 (C-6), 167.3 (C-7), 92.8 (C8), 158.3 (C-9), 105.6 (C-10), $122.6\left(\mathrm{C}-1^{\prime}\right), 128.9\left(\mathrm{C}-2^{\prime}\right)$, $113.2\left(\mathrm{C}-3^{\prime}\right), 159.5\left(\mathrm{C}-4^{\prime}\right), 113.9\left(\mathrm{C}-5^{\prime}\right), 128.9\left(\mathrm{C}-6^{\prime}\right), 55.8$ (C-11), 55.9 (C-12).

3.2. Determination of the Blood Glucose Concentration Using P. major L. Extracts in In Vivo Study. From Figure 3 and Table 4, the blood glucose levels of obese mice in the nontreated group Cage 1 (NCd) showed no decrease throughout the 15-day treatment but significantly decreased in the mice that were treated with Glucophage (Cage 2), up to $55.7 \%$ only after 3 days of treatment in comparison with the controls at the same time $(p<0.05)$. In the groups that were treated with $P$. major L. extracts EP (Cage 3), EP3 fraction (Cage 4), EP4 fraction (Cage 5), and EP 6 fraction (Cage 6) with a dose of $400 \mathrm{mg} / \mathrm{kg}$, in general, we also observed a significant decrease in blood glucose level after $2 \mathrm{~h}, 4 \mathrm{~h}, 8 \mathrm{~h}, 3$ days, and 15 days $(p<0.05)$. A substantial decrease was recorded in groups treated with extract EP4 (Cage 5) and EP 6 (Cage 6). With EP4 treatment (Cage 5), the blood glucose dropped quickly after the first few hours of oral intake and showed a $39.1 \%$ and $51.51 \%$ decrease compared to the controls $(p<0.01)$ after 3 days and 5 days, respectively. After 3 days of EP6 treatment (Cage 6), the blood glucose levels in this group dropped as low as $65.72 \%$ $(p<0.01)$ and stabilized during the rest of the experiment.

Obese mice have high levels of cholesterol and triglycerides. Based on Table 5, mice that received the treatment with EP6 extract (Cage 6) have a significantly higher level of cholesterol and triglycerides $(2.00 \mathrm{mmol} / \mathrm{L}, 1.25 \mathrm{mmol} / \mathrm{L})$ compared with those of the diabetic obese mice with no treatment (Cage 1-NCd) $(1.22 \mathrm{mmol} / \mathrm{L}, 0.83 \mathrm{mmol} / \mathrm{L})$ but lower than those of the obese mice (NCf) $(2.91 \mathrm{mmol} / \mathrm{L}$, $1.99 \mathrm{mmol} / \mathrm{L})$. High AST and ALT levels are reasonably sensitive indicators of liver damage. In the normal mice
(NC) group, AST and ALT levels were 21.9(U/L) and 11.85 (U/L), respectively. In the treated group (cage 6), those levels were 23.1 (U/L) and 26.5 (U/L) (AST, ALT). However, those levels were significantly lower than those of the diabetic group without treatment (Cage 1), $100.02(\mathrm{U} / \mathrm{L})$, and 99.59 (U/L) (AST, ALT).

\section{Discussion}

The STZ administration to damage the pancreas in obese mice led to an increase in their blood sugar levels. The condition accompanied by high consumption of food and water indicates signs of diabetes in mice $[18,19]$. In this study, the use of selective fractions and crude extract from $P$. major L. to control blood sugar levels of obese mice with type 2 diabetes showed positive results. Fraction EP6 showed the ability to reduce blood glucose in type 2 diabetes obese mice by $67.73 \%$ due to the presence of two flavonoid compounds: 7-O-methylapigenin (EPL7) and 7, 4'-Odimethylapigenin (EPL8). This result was supported by previous research where the glycemic control effect of $P$. major L. extracts was contributed due to the presence of flavonoids compounds. The characteristic structures of these compounds contain aromatic rings with hydroxyl groups and are similar to standard flavonoid compounds used for antioxidants or inhibiting $\alpha$-glucosidase activity, such as quercetin and resveratrol [20-24]. The efficacy of EP6 fraction is comparable to the result obtained from the treatment with Glucophage (a standard antihyperglycemic drug currently being used to treat type 2 diabetes patients) in the positive control group after 15 days. This demonstrates that $P$. major L. extracts can reduce the blood glucose level in type 2 diabetic mice. In an oral glucose tolerance test aiming at measuring the efficacy to eliminate excess glucose after an oral glycemic load [9], fasting mice were given a $500 \mathrm{mg} / \mathrm{kg}$ dose of $P$. major L. extract before the administration of glucose $1.25 \mathrm{~g} / \mathrm{kg}$ orally. It was observed that there is a $27 \%$ reduction in blood glucose in diabetic mice given the extract at 120 minutes compared to the nontreated group. Unfortunately, the blood glucose levels were only measured at 30, 60,90 , and 120 minutes, so there is not enough information to conclude about the long-term antiglycemic effect of the $P$. major L. extract. The research, however, provided valuable results when a glucose test was carried out on normal mice. Even within the normal nondiabetic group, those given $P$. major L. extract have lowered blood glucose to normal ones without treatment (a 23\% reduction) and this level is comparable to normal ones given glibenclamide, a 
TABLE 5: Comparison of blood glucose level, cholesterol level, triglycerides level, and liver function level (AST, ALT) between diabetic mice treated with EP6 (Cage 6), diabetic mice without any treatment (Cage 1), overweight mice (NCf), and normal mice (NC).

\begin{tabular}{lccccc}
\hline & Glucose $(\mathrm{mmol} / \mathrm{L})$ & Cholesterol $(\mathrm{mmol} / \mathrm{L})$ & Triglycerides $(\mathrm{mmol} / \mathrm{L})$ & AST $(\mathrm{U} / \mathrm{L})$ & ALT $(\mathrm{U} / \mathrm{L})$ \\
\hline Cage 6 & 11.01 & 2.0 & 1.25 & 23.10 & 26.50 \\
Cage 1 & 25.71 & 1.22 & 0.83 & 100.02 & 99.59 \\
NCf & 11.50 & 2.91 & 1.99 & 25.91 \\
NC & 5.620 & 0.71 & 0.72 & 21.90 \\
\hline
\end{tabular}

commonly used prescription in diabetes mellitus type 2 (a $26 \%$ reduction compared to normal ones without treatment). This supports our result that extract from P. major $L$. could contain chemicals for reducing blood glucose in diabetes mellitus. In a thorough 14-day experiment on the antiglycemic effect of $P$. major L. extracts in mice [7], a dose of $1000 \mathrm{mg} / \mathrm{kg}$ was able to reduce blood glucose in diabetic mice by $47 \%$ compared to the nontreated group after 5 -day treatment. This result was followed by a $43 \%$ reduction on the $5^{\text {th }}$ day and a $54 \%$ reduction on the $14^{\text {th }}$ day. In comparison, our P. major L. extract, used at a dose of $400 \mathrm{mg} / \mathrm{kg}$, showed a $65 \%$ reduction in blood glucose of the diabetic mice compared to the nontreated group after 3 days of treatment and an almost 70\% reduction ensuing 15-day treatment. Clearly, our extract shows improvement in the dosage used and time to take effect. We hypothesize that the oral intake of the extracts can enhance the capacity of $\beta$-cell to upregulate insulin synthesis or trigger the renewal of $\beta$-cell or preservation of pancreatic $\beta$-cell mass as indicated in other research $[25,26]$. This leads to improved glycemic control and cellular glucose uptake. Still, more quantitative investigation on the morphology of pancreatic islets needs to be done to check if islets were restored after using P. major L. extracts and led to the restoration of plasma glucose and insulin level in diabetic mice. As a consequence, serum insulin levels also need to be checked to confirm this effect. However, these experiments are beyond the scope of this paper. Meanwhile, we did assess biochemical parameters on diabetic mice after 15 days of treatment with EP6 fraction and compared with other groups of mice. There was no improvement in cholesterol and triglycerides within the group given EP6 extract, so it is possible that EP6 extract had no effect on fat metabolism [27, 28]. However, differences in AST and ALT levels among those groups are observed. Abnormal changes in AST and ALT in the blood are indicators of liver damage due to intoxication or pathology, which was reported in studies using STZ on mouse models leading to the damage of the pancreas and liver. $[29,30]$. The AST and ALT levels of the EP6-treated group were significantly lower than the nontreated diabetic mice (Cage 1), slightly less than the nontreated and nondiabetic obese mice (NCf) and comparable to normal mice. Thus, it is possible that EP6 extract improved liver function, reducing elevated liver enzymes in diabetic mice to a similar level of AST in the control group (NC).

\section{Conclusion}

The present research indicates the potential of $P$. major L. to control the blood sugar of type 2 diabetes in obese mice at a lowered dose and shorter time to take effect compared to previous research. We have provided thorough isolation and bioactive structural determination of $P$. major $\mathrm{L}$. extracts. The in vivo study also pointed out the efficacy of fraction EP6 in reducing blood glucose to a level comparable to that obtained from Glucophage treatment. Two components of EP6 fraction were also identified, 7-O-methylapigenin (EPL7) and 7, 4'-O-dimethylapigenin (EPL8). Fortunately, EP6 does not show any damage to liver functions as usually observed in other diabetic treatments. Further studies on the glycemic regulating mechanism of $P$. major L. extract via the release of pancreatic insulin should be considered by tracking the insulin level in the bloodstream and urine excretion.

\section{Data Availability}

The data used to support the findings of this study are included within the article.

\section{Conflicts of Interest}

The authors declare that there are no conflicts of interest.

\section{Acknowledgments}

This research was funded by Vietnam National Foundation for Science and Technology Development (NAFOSTED) under Grant no. 104.01-2017.332.

\section{References}

[1] E. Saedi, M. R. Gheini, F. Faiz, and M. A. Arami, "Diabetes mellitus and cognitive impairments," World Journal of Diabetes, vol. 7, no. 17, p. 412, 2016.

[2] F. L. Fimognari, R. Pastorelli, and R. A. Incalzi, "Phenformininduced lactic acidosis in an older diabetic patient: a recurrent drama (phenformin and lactic acidosis)," Diabetes Care, vol. 29, no. 4, pp. 950-951, 2006.

[3] L. F. Verdonck, B. Sangster, A. N. P. Van Heijst, G. De Groot, and R. A. A. Maes, "Buformin concentrations in a case of fatal lactic acidosis," Diabetologia, vol. 20, no. 1, pp. 45-46, 1981.

[4] Anti-Diabetes Medication to Be Taken off the Market, European Medicines Agency, Amsterdam, Netherlands, 2010.

[5] A. M. Lincoff, K. Wolski, S. J. Nicholls, and S. E. Nissen, "Pioglitazone and risk of cardiovascular events in patients with type 2 diabetes mellitus," JAMA, vol. 298, no. 10, pp. 1180-1188, 2007.

[6] J. Jamaluddin, A. A. Sharifa, and N. R. S. A. Sharifah, "GC-MS analysis of various extracts from leaf of Plantago major used as traditional medicine," World Applied Sciences Journal, vol. 17, 2012. 
[7] M. A. Abdulghani, I. Hamid, R. A. Al-Naggar, and M. T. Osman, "Potential antidiabetic activity of Plantago major leaves extract in Streptozocin-induced diabetic mice," Research Journal of Pharmaceutical, Biological and Chemical Sciences, vol. 5, no. 2, pp. 896-902, 2014.

[8] H. Oor, M. Juing, B. Chee, and B. L. Kuehandzolkepliothman, "Medicinal properties of Plantago major: hypoglycaemic and male fertility studies," Pertanika Journal of Tropical Agricultural Science, vol. 23, no. 1, pp. 29-35, 2000.

[9] M. J. S. Benjamin, M. Davis, and G. F. Rall, "Hypoglycemic effect due to insulin stimulation with Plantago major in wistar mice," Medicinal \& Aromatic Plants (Los Angeles), vol. 176, no. 1, pp. 139-148, 2017.

[10] P. Gürbüz, L. Ö. Demirezer, Z. Güvenalp, A. Kuruüzüm-Uz, and C. Kazaz, "Isolation and structure elucidation of uncommon secondary metabolites from cistus salviifolius L," Records of Natural Products, vol. 9, no. 2, pp. 175-183, 2015.

[11] M. H. Abd El-Razek, "NMR assignments of four catechin epimers," Asian Journal of Chemistry, vol. 19, no. 6, pp. 4867-4872, 2007.

[12] A. Hisham, G. J. Kumar, Y. Fujimoto, and N. Hara, "Salacianone and salacianol, two triterpenes from Salacia beddomei," Phytochemistry, vol. 40, no. 4, pp. 1227-1231, 1995.

[13] J. A. Sánchez-Burgos, M. V. Ramírez-Mares, J. A. GallegosInfante et al., "Isolation of lupeol from white oak leaves and its anti-inflammatory activity," Industrial Crops and Products, vol. 77, pp. 827-832, 2015.

[14] J. Dou, J. D. McChesney, R. D. Sindelar, D. K. Goins, I. A. Khan, and L. A. Walker, "A new quassinoid from crude quassin-extract of Quassia amara," International Journal of Pharmacognosy, vol. 34, no. 5, pp. 349-354, 1996.

[15] A. J. King, "The use of animal models in diabetes research," British Journal of Pharmacology, vol. 166, no. 3, pp. 877-894, 2012.

[16] N. T. Quan, Create a Diabetic Model on Mice and Investigate the Ability to Control Blood Glucose Level from Some Natural Products, VNU University of Science, Hanoi, Vietnam, 2009.

[17] C. Kumar, R. Kumar, and S. Nehar, "Induction of type-II diabetes by high fructose diet and low dose of intraperitoneal injection of streptozotocin in albino mice," International Journal for Pharmaceutical Research Scholars (IJPRS), vol. 3, pp. 671-677, 2014.

[18] M. Zafar and S. N.-H. Naqvi, "Effects of STZ-induced diabetes on the relative weights of kidney, liver and pancreas in albino mice: a comparative study efectos de la diabetes inducida por STZ en los pesos relativos de Riñón, Hígado y Páncreas en Ratas Albinas: un Estudio comparativo," International Journal of Morphology, vol. 28, no. 1, pp. 135-142, 2010.

[19] B. L. Furman, "Streptozotocin-induced diabetic models in mice and mice," Current Protocols in Pharmacology, vol. 70, no. 1, 2015.

[20] M. B. Adom, M. Taher, M. F. Mutalabisin et al., "Chemical constituents and medical benefits of Plantago major," Biomedicine \& Pharmacotherapy, vol. 96, pp. 348-360, 2017.

[21] I. Kobeasy, M. Abdel-Fatah, S. M. Abd El-Salam, and Z. M. ElOla Mohamed, "Biochemical studies on Plantago major L. and Cyamopsis tetragonoloba L," International Journal of Biodiversity and Conservation, vol. 3, no. 3, pp. 83-91, 2011.

[22] M. N. Sarian, Q. U. Ahmed, S. Z. Mat So'ad et al., "Antioxidant and antidiabetic effects of flavonoids: a structureactivity relationship based study," BioMed Research International, vol. 2017, Article ID 8386065, 2017.

[23] M. Hawash, N. Jaradat, J. Elaraj, A. Hamdan, S. A. Lebdeh, and T. Halawa, "Evaluation of the hypoglycemic effect of seven wild folkloric edible plants from palestine (antidiabetic effect of seven plants from palestine)," Journal of Complementary and Integrative Medicine, vol. 17, no. 1, pp. 1-10, 2020.

[24] R. K. Al-Ishaq, M. Abotaleb, P. Kubatka, K. Kajo, and D. Büsselberg, "Flavonoids and their anti-diabetic effects: cellular mechanisms and effects to improve blood sugar levels," Biomolecules, vol. 9, no. 9, 2019.

[25] A. Noor, S. Gunasekaran, and M. A. Vijayalakshmi, "Improvement of insulin secretion and pancreatic $\beta$-cell function in streptozotocin-induced diabetic mice treated with aloe vera extract," Pharmacognosy Research, vol. 9, no. Suppl 1, pp. S99-S104, 2017.

[26] Y. S. Oh, "Plant-derived compounds targeting pancreatic beta cells for the treatment of diabetes," Evidence-Based Complementary and Alternative Medicine, vol. 2015, Article ID 629863, 2015.

[27] M. Marrelli, F. Conforti, F. Araniti, and G. Statti, "Effects of saponins on lipid metabolism: a review of potential Health benefits in the treatment of obesity," Molecules, vol. 21, no. 10, p. 1404, 2016.

[28] C. Roh and U. Jung, "Screening of crude plant extracts with anti-obesity activity," International Journal of Molecular Sciences, vol. 13, no. 2, pp. 1710-1719, 2012.

[29] X.-J. Huang, Y.-K. Choi, H.-S. Im, O. Yarimaga, E. Yoon, and H.-S. Kim, "Aspartate aminotransferase (AST/GOT) and alanine aminotransferase (ALT/GPT) detection techniques," Sensors, vol. 6, no. 7, pp. 756-782, 2006.

[30] M. N. A Takahashi, C. Sekiya, Y. Yazaki et al., "Hepatic GOT and GPT activities in patients with various liver diseases-especially alcoholic liver disease," Hokkaido Igaku Zasshi, vol. 61, no. 3, pp. 431-436, 1986. 